\title{
Intermediate-Generalized Chaplygin Gas inflationary universe model
}

\author{
Ramón Herrera $*$ Marco Olivares $t \dagger$ and Nelson Videlat \\ Instituto de Física, Pontificia Universidad Católica de Valparaíso, \\ Avenida Brasil 2950, Casilla 4059, Valparaíso, Chile.
}

(Dated: December 10, 2017)

\begin{abstract}
An intermediate inflationary universe model in the context of a generalized Chaplygin gas is considered. For the matter we consider two different energy densities; a standard scalar field and a tachyon field, respectively. In general, we discuss the conditions of an inflationary epoch for these models. We also, use recent astronomical observations from Wilkinson Microwave Anisotropy Probe seven year data for constraining the parameters appearing in our models.
\end{abstract}

PACS numbers: $98.80 . \mathrm{Cq}$

\footnotetext{
*Electronic address: ramon.herrera@ucv.cl

†Electronic address: marco.olivares@ucv.cl

${ }^{\ddagger}$ Electronic address: nelson.videla@ucv.cl
} 


\section{INTRODUCTION}

The inflationary universe was introduced [1, 2] as a manner of addressing pressing problems (horizon, flatness, monopoles, etc.) that were eating away at the bases of the otherwise rather prosperous Big-Bang model. The most significant feature of the inflationary universe model is that it provides a causal interpretation of the origin of the observed anisotropy of the cosmic microwave background radiation (CMB) and the structure formation in the universe[3, 4]].

Exact solutions exist for power-law and de-Sitter inflationary universes and they are created by exponential and constant scalar potentials, see Ref.[1, 5]. Exact solutions can also be obtained for the scenario of intermediate inflation, where the scale factor, $a(t)$, increases as

$$
a=\exp \left[A t^{f}\right]
$$

in which $A$ and $f$ are two constants; $A>0$ and $0<f<1$ [6]. The expansion of this inflationary scenario is slower than de-Sitter inflation, but faster than power law inflation, this is the denotation why it is called "intermediate". The intermediate model was originally formulated as an exact solution, but it may be best inspired from the slow-roll approximation. From the slow-roll approximation, it is possible to have a spectrum of density perturbations which presents a spectral index $n_{s} \sim 1$ and also in particular $n_{s}=1$ (Harrizon-Zel'dovich spectrum) for the value $f=2 / 3$ [7]. However, the value $n_{s}=1$ is disfavored by the current Wilkinson Microwave Anisotropy Probe (WMAP) observational data[3, 4]. Also, the tensor perturbations which could be present in this model, through of the parametrized by the tensor to scalar ratio $r$, which is significantly $r \neq 0[8,9]$. On the other hand, the motivation to study this expansion becomes from string/M-theory, indicates that in order to have a ghost-free action high order curvature invariant corrections to the Einstein-Hilbert action must be relative to the Gauss-Bonnet (GB) term[10], where this expansion appears to the low-energy string effective action[11, 12] (see also, Ref.[13]).

On the other hand, the generalized Chaplygin gas (GCG) is other aspirant for explaining the acceleration of universe. The exotic equation of state of the GCG is given by [14]

$$
p_{C h}=-\frac{\alpha}{\rho_{C h}^{\beta}}
$$

where $\rho_{C h}$ and $p_{C h}$ are the energy density and pressure of the GCG, $\beta$ is a constant in 
which $\beta \leq 1$, and $\alpha$ is a positive constant. In particular, when $\beta=1$ corresponds to the original Chaplygin gas [14]. Replacing, Eq.(21) into the stress-energy conservation equation, the energy density results

$$
\rho_{C h}=\left[\alpha+\frac{B}{a^{3(1+\beta)}}\right]^{\frac{1}{1+\beta}}=\rho_{C h 0}\left[B_{s}+\frac{\left(1-B_{s}\right)}{a^{3(1+\beta)}}\right]^{\frac{1}{1+\beta}} .
$$

Here, $a$ is the scale factor and B is a positive integration constant. In this way, the GCG is characterized by two parameters, $B_{s}=\alpha / \rho_{C h 0}^{1+\beta}$ and $\beta$. These parameter has been confronted by observational data, see Refs.[15, 16]. In particular, the values of $B_{s}=0.73_{-0.06}^{+0.06}$ and $\beta=-0.09_{-0.12}^{+0.15}$ was obtained in Ref.[16]. Also, in Ref.[17] the values $0.81 \lesssim B_{s} \lesssim 0.85$ and $0.2 \lesssim \beta \lesssim 0.6$ were found from the observational data arising from Archeops for the location of the first peak, BOOMERANG for the location of the third peak, supernova and high-redshift observations. Recently, the values of $B_{s}=0.775_{-0.0161-0.0338}^{+0.0161+0.037}$ and $\beta=$ $0.00126_{-0.00126-0.00126}^{+0.000970+0.00268}$ was obtained from Markov Chain Monte Carlo method[18].

The Chaplygin gas arises as an effective fluid of a generalized d-brane the space time, in a Born-Infeld action [17] and these models have been extensively analyzed in Ref.[19]. In the model of Chaplygin inspired in an inflationary scenario commonly the standard scalar field drives inflation, in which the energy density given by Eq.(3), can be extrapolate in the Friedmann equation for archiving an appropriate inflationary period [20]. However, also a tachyonic field in a Chaplygin inflationary universe model was considered in Ref.[21]. The possibility of having Chaplygin models with scalar field and tachyon field has been considered in Ref.[22]. The modification of the Friedmann equation is realized from an extrapolation of Eq.(3), where we identifying the density matter with the contributions of the density energy associated to the standard scalar field or tachyonic field [17, 21]. In this way, the GCG model may be viewed as a variation of gravity and there has been great interest in the elaboration of early universe scenarios motivated by string/M-theory[23]. It is well known that these modifications can lead to significant changes in the early universe.

In this paper we would like to study intermediate-GCG inflationary universe model in which different types of energy densities are taken into account. In particular, (i) when the energy density is a standard scalar field, and (ii) when the energy density is a tachyon field. We will investigate the dynamic in both models and also we shall utilize to the seven-year data WMAP to restrict the parameters in our models. The outline of the paper is as follows. The next section presents the dynamic of the intermediate-GCG Inflationary scenario for our 
two models. Section III deals with the calculations of cosmological perturbations. Finally, in Sect $[\mathrm{IV}$ we conclude with our finding.

\section{INTERMEDIATE-GCG INFLATIONARY UNIVERSE MODEL}

It is well know that the GCG model can also be used to describe the early universe. During inflation the gravity dynamics may give rise to a modified Friedmann equation[17]

$$
H^{2}=\frac{\kappa}{3}\left(\alpha+\rho_{\phi}^{1+\beta}\right)^{\frac{1}{1+\beta}}
$$

where $\kappa=8 \pi / m_{p}^{2}, m_{p}$ is the reduced Planck mass, $\rho_{\phi}$ is the energy density of the scalar field and $H=\dot{a} / a$ is the Hubble parameter. This modification in the Friedmann equation is the so-called Chaplygin inspired inflation scenario[17]. Following this idea, lately, some work has been done, this involves Chaplygin inflationary universe model, see Refs. [21, 24].

In the following, we will considers two matter fields for $\rho_{\phi}$; the standard scalar field and tachyonic field, respectively. For convenience we use units in which $c=\hbar=1$.

\section{A. Standard scalar field}

We consider that the matter content of the universe is a standard scalar field $\phi$, in which the energy density is given by $\rho_{\phi}=\frac{\dot{\phi}^{2}}{2}+V(\phi)$ and the pressure $p_{\phi}=\frac{\dot{\phi}^{2}}{2}-V(\phi)$ where $V(\phi)=V$ is the scalar potential. The conservation equation is given by

$$
\dot{\rho}_{\phi}+3 H\left(\rho_{\phi}+p_{\phi}\right)=0
$$

which is equivalent to the equation of motion of the standard scalar field

$$
\ddot{\phi}+3 H \dot{\phi}+V^{\prime}=0
$$

in which, $V^{\prime}=\partial V(\phi) / \partial \phi$ and the dots mean derivatives with respect to time.

From Eqs.(41) and (5), we get

$$
\dot{\phi}^{2}=\frac{2}{\kappa}(-\dot{H})\left[1-\alpha\left(\frac{\kappa}{3 H^{2}}\right)^{1+\beta}\right]^{\frac{-\beta}{1+\beta}}
$$

and the effective potential becomes

$$
V=\frac{3}{\kappa} H^{2}\left[1-\alpha\left(\frac{\kappa}{3 H^{2}}\right)^{1+\beta}\right]^{\frac{1}{1+\beta}}+\frac{1}{\kappa} \dot{H}\left[1-\alpha\left(\frac{\kappa}{3 H^{2}}\right)^{1+\beta}\right]^{\frac{-\beta}{1+\beta}} .
$$


Note that for $\alpha=0$, the expression for $\dot{\phi}^{2}$ and the scalar potential $V$ given by Eqs.(7) and (8), reduced to typical expression corresponding to standard inflation, where $\dot{\phi}^{2}=-2 \dot{H} / \kappa$ and $V=\left(3 H^{2}+\dot{H}\right) / \kappa[7]$.

The solution for the standard scalar field $\phi$, using Eqs.(1) and (17) is given by

$$
\phi(t)-\phi_{0}=\frac{\mathcal{B}[t]}{K}
$$

where $\phi(t=0)=\phi_{0}$ is an integration constant, the constant $K \equiv \sqrt{6}(1+$ $\beta)\left(\frac{\kappa}{3}\right)^{\frac{2-f}{4(1-f)}} \sqrt{(1-f)}(A f)^{\frac{-1}{2(1-f)}} \alpha^{\frac{1}{4(1-f)(1+\beta)}}$ and

$$
\mathcal{B}[t] \equiv B\left[\left(\frac{\kappa}{3}\right)^{1+\beta} \frac{\alpha t^{2(1-f)(1+\beta)}}{(A f)^{2(1+\beta)}} ; \frac{f}{2(1-f)(1+\beta)}, \frac{2+\beta}{2(1+\beta)}\right] .
$$

Here, $\mathcal{B}[t]$, is the incomplete Beta function [25] and without loss of generality $\phi_{0}=0$.

For the Hubble parameter $H(\phi)$, we get $H(\phi)=A f\left(\mathcal{B}^{-1}[K \phi]\right)^{f-1}$, where $\mathcal{B}^{-1}$ represent the inverse function of the incomplete Beta function.

In the slow-roll approximation, the first term of Eq.(8) dominate the effective potential at large value of $\phi$ and using Eqs.(8) and (9), we have

$$
V(\phi)=\left[\left(\frac{3 A^{2} f^{2}}{\kappa}\right)^{1+\beta}\left(\mathcal{B}^{-1}[K \phi]\right)^{-2(1-f)(1+\beta)}-\alpha\right]^{\frac{1}{1+\beta}} .
$$

Note that we would have obtained the same potential $V(\phi)$ represented by Eq.(10), considering the set of slow-roll conditions, where $\dot{\phi}^{2} \ll V(\phi)$ and $\ddot{\phi} \ll 3 H \dot{\phi}$.

The dimensionless slow-roll parameters in this case become $\varepsilon \equiv-\frac{\dot{H}}{H^{2}}=\frac{1-f}{A f\left(\mathcal{B}^{-1}[K \phi]\right)^{f}}$, and $\eta \equiv-\frac{\ddot{H}}{H \dot{H}}=\frac{2-f}{A f\left(\mathcal{B}^{-1}[K \phi]\right)^{f}}$. The inflationary scenario takes place when the slow-roll parameter $\varepsilon<1$ or analogously when $\ddot{a}>0$. Therefore, the condition for inflation to occur is satisfied when the standard field $\phi>\frac{1}{K} \mathcal{B}\left[\left(\frac{1-f}{A f}\right)^{1 / f}\right]$.

Using Eq.(9), the number of e-folds $N$ between two values of cosmological times $t_{1}$ and $t_{2}$ or analogously between two different values of $\phi$, in which $\phi\left(t=t_{1}\right)=\phi_{1}$ and $\phi\left(t=t_{2}\right)=\phi_{2}$, becomes

$$
N=\int_{t_{1}}^{t_{2}} H d t=A\left[\left(t_{2}\right)^{f}-\left(t_{1}\right)^{f}\right]=A\left[\left(\mathcal{B}^{-1}\left[K \phi_{2}\right]\right)^{f}-\left(\mathcal{B}^{-1}\left[K \phi_{1}\right]\right)^{f}\right]
$$

Considering that the inflationary scenario begins at the earliest possible scenario in which $\varepsilon=1[9]$, then the scalar field $\phi_{1}$, is given by $\phi_{1}=\frac{1}{K} \mathcal{B}\left[\left(\frac{1-f}{A f}\right)^{1 / f}\right]$. 


\section{B. Tachyon field}

For the case of the tachyonic field, the energy density and the pressure are given by

$\rho_{\phi}=\frac{V(\phi)}{\sqrt{1-\dot{\phi}^{2}}}$ and $P_{\phi}=-V(\phi) \sqrt{1-\dot{\phi}^{2}}$, respectively. Here, $\phi$ represents the tachyon field and $V(\phi)=V$ is the tachyonic potential. The equation of motion for the tachyonic field from Eq.(5) , is given by

$$
\frac{\ddot{\phi}}{1-\dot{\phi}^{2}}+3 H \dot{\phi}+\frac{V^{\prime}}{V}=0 .
$$

Using, Eqs.(4) and (12), we get

$$
\dot{\phi}^{2}=-\frac{2 \dot{H}}{3 H^{2}}\left[1-\alpha\left(\frac{\kappa}{3 H^{2}}\right)^{1+\beta}\right]^{-1},
$$

and the tachyonic potential as function of the Hubble parameter $H$ and $\dot{H}$, becomes

$$
V=\left[\left(\frac{3}{\kappa}\right)^{1+\beta} H^{2(1+\beta)}-\alpha\right]^{\frac{1}{1+\beta}} \sqrt{1+\frac{2 \dot{H}}{3 H^{2}}\left[1-\alpha\left(\frac{\kappa}{3 H^{2}}\right)^{1+\beta}\right]^{-1}} .
$$

Again, when $\alpha=0$ the expressions for the velocity of the tachyonic field $\dot{\phi}$ and $V$ reduced to standard tachyonic model, where $\dot{\phi}=\sqrt{-2 \dot{H} /\left(3 H^{2}\right)}$ and $V=(3 / \kappa) H^{2} \sqrt{1+2 \dot{H} /\left(3 H^{2}\right)}$ (see Ref.[26]).

From Eqs.(1) and (13), the solution for the tachyonic field becomes

$$
\phi(t)-\phi_{0}=\frac{\widetilde{\mathcal{B}}[t]}{\widetilde{K}},
$$

where the constant $\widetilde{K} \equiv \frac{\sqrt{6}(1+\beta) \sqrt{1-f}\left(\frac{\kappa}{3}\right)^{\frac{2-f}{4(1-f)}} \alpha^{\frac{2-f}{4(1+\beta)(1-f)}}}{(A f)^{\frac{1}{2(1-f)}}}$, and

$$
\widetilde{\mathcal{B}}[t] \equiv B\left[\left(\frac{\kappa}{3}\right)^{1+\beta} \frac{\alpha t^{2(1+\beta)(1-f)}}{(A f)^{2(1+\beta)}} ; \frac{2-f}{4(1+\beta)(1-f)}, \frac{1}{2}\right] .
$$

Here, again $\widetilde{\mathcal{B}}$ is the incomplete Beta function. Now, by using Eqs.(1) and (15), the Hubble parameter as a function of the tachyon field, becomes $H(\phi)=A f\left(\widetilde{\mathcal{B}}^{-1}[\widetilde{K} \phi]\right)^{f-1}$, where $\widetilde{\mathcal{B}}^{-1}$ represent the inverse function of the incomplete Beta function and $\phi_{0}=0$.

Analogously, as the case of the standard scalar field, during the slow-roll approximation, the first term of Eq.(14) dominate the effective potential at large value of $\phi$ and from Eqs.(1) and (15), we get

$$
V(\phi)=\left[\left(\frac{3}{\kappa}\right)^{1+\beta} \frac{(A f)^{2(1+\beta)}}{\left(\left(\widetilde{\mathcal{B}}^{-1}[\widetilde{K} \phi]\right)^{1-f}\right)^{2(1+\beta)}}-\alpha\right]^{\frac{1}{1+\beta}}
$$


In addition, note that again we would have obtained the same tachyonic potential, considering the set of slow-roll conditions for the tachyonic field, where $\dot{\phi}^{2} \ll 1$ and $\ddot{\phi} \ll 3 H \dot{\phi}$.

Again, as before now we can write the dimensionless slow-roll parameters for the case of the tachyonic field. Considering Eqs.(1) and (15), we get $\varepsilon=\frac{1-f}{A f}\left(\widetilde{\mathcal{B}}^{-1}[\widetilde{K} \phi]\right)^{-f}$, and $\eta=\frac{2-f}{A f}\left(\widetilde{\mathcal{B}^{-1}}[\widetilde{K} \phi]\right)^{-f}$.

The number of e-folds between times $t_{1}$ and $t_{2}$ using Eq.(15) is given by

$$
N=\int_{t_{1}}^{t_{2}} H d t=A\left[\left(t_{2}\right)^{f}-\left(t_{1}\right)^{f}\right]=A\left[\left(\widetilde{\mathcal{B}}^{-1}\left[\widetilde{K} \phi_{2}\right]\right)^{f}-\left(\widetilde{\mathcal{B}}^{-1}\left[\widetilde{K} \phi_{1}\right]\right)^{f}\right]
$$

Analogously, as the case of the standard field, the inflation begins at the earliest possible scenario, in which $\phi_{1}=\frac{1}{\widetilde{K}} \widetilde{\mathcal{B}}\left[\left(\frac{1-f}{A f}\right)^{1 / f}\right]$.

\section{COSMOLOGICAL PERTURBATIONS}

In this section we will analyze the scalar and tensor perturbations for our models, where the matter content of the universe are the standard scalar field and the tachyonic field, respectively.

\section{A. Standard scalar field}

In the following, we will consider the power spectra of scalar and tensor perturbations to the metric in Chaplygin inflation. We introduce the gauge invariant quantity [27, 28]

$$
\zeta=H+\frac{\delta \rho}{\dot{\rho}}
$$

where $\psi$ is the gravitational potential. On slices of uniform density $\zeta$ reduces to the curvature perturbation. A fundamental characteristic attribute of $\zeta$ is that it is nearly constant on super-horizon scales. This feature, result to be a consequence of stress-energy conservation and does not depend on the gravitational dynamics|29] (see also, Ref.[30]). In this context, it continues unchanged in Chaplygin inflation[20, 31]. In this form, the power spectrum related to curvature spectrum, could be written as $\mathcal{P}_{\mathcal{R}} \simeq\left\langle\zeta^{2}\right\rangle$. It can be shown that on super-horizon scales, the curvature perturbation on slices of uniform density is equivalent to the comoving curvature perturbation. Therefore, for the spatially flat gauge, we have $\zeta=H \frac{\delta \phi}{\phi}$, in which $|\delta \phi|=H / 2 \pi[32]$. 
In this way, the power spectrum considering Eq.(17), is given by

$$
\mathcal{P}_{\mathcal{R}} \simeq \frac{\kappa}{8 \pi^{2}} H^{4}(-\dot{H})^{-1}\left[1-\alpha\left(\frac{\kappa}{3 H^{2}}\right)^{1+\beta}\right]^{\frac{\beta}{1+\beta}},
$$

or equivalently in terms of the standard scalar field $\phi$

$$
\mathcal{P}_{\mathcal{R}} \simeq \frac{\kappa}{8 \pi^{2}} \frac{(A f)^{3}}{1-f}\left(\mathcal{B}^{-1}[K \phi]\right)^{-(2-3 f)}\left[1-\alpha\left(\frac{\kappa}{3 A^{2} f^{2}}\right)^{1+\beta}\left(\mathcal{B}^{-1}[K \phi]\right)^{2(1-f)(1+\beta)}\right]^{\frac{\beta}{1+\beta}} .
$$

The power spectrum $\mathcal{P}_{\mathcal{R}}$, also can be expressed in terms of the number of e-folds $N$, as

$$
\mathcal{P}_{\mathcal{R}}=\frac{\kappa}{8 \pi^{2}} \frac{(A f)^{3}}{1-f}\left[\frac{A f}{1+f(N-1)}\right]^{\frac{2-3 f}{f}}\left[1-\alpha\left(\frac{\kappa}{3 A^{2} f^{2}}\right)^{1+\beta}\left[\frac{1+f(N-1)}{A f}\right]^{\frac{2(1-f)(1+\beta)}{f}}\right]^{\frac{\beta}{1+\beta}} .
$$

Numerically from Eq.(20) we obtained a constraint for the parameter $A$. In fact, we can obtain the value of the parameter $A$ for given values of $f, \alpha$ and $\beta$ parameters when number $N$ and the power spectrum $\mathcal{P}_{\mathcal{R}}$ are given. In particular, for the values $\mathcal{P}_{\mathcal{R}}=2.4 \times 10^{-9}$, $N=60, f=1 / 2$ and $\kappa=1$, we obtained that for the pair $(\alpha=0.775, \beta=0.00126)$ [18], which corresponds to the parameter $A \simeq 8.225 \times 10^{-2}$, for the pair $(\alpha=0.81, \beta=0.2)[17]$, corresponds to $A \simeq 2.635 \times 10^{-2}$ and for the pair $(\alpha=0.85, \beta=0.6)$ [17], which corresponds to $A \simeq 8.407 \times 10^{-5}$.

On the other hand, the scalar spectral index $n_{s}$ is given by the expression $n_{s}=$ $d \ln \mathcal{P}_{\mathcal{R}} / \ln k$ and considering Eq.(19), we get

$$
n_{s} \simeq 1-\frac{2-3 f}{A f\left(\mathcal{B}^{-1}[K \phi]\right)^{f}}-\left[\frac{\alpha_{0} \beta \gamma}{A f(1+\beta)}\right]\left[1-\alpha_{0}\left(\mathcal{B}^{-1}[K \phi]\right)^{\gamma}\right]^{-1}\left(\mathcal{B}^{-1}[K \phi]\right)^{\gamma-f},
$$

where the constants $\alpha_{0}=\alpha\left(\frac{\kappa}{3 A^{2} f^{2}}\right)^{1+\beta}$ and $\gamma=2(1-f)(1+\beta)$, respectively.

From Eq.(21), we clearly see that $n_{s} \neq 1$, for $f=2 / 3$ (recall that $1>f>0$ ). However, as occurs in Ref.[9], $n_{s}=1$ for the value $f=2 / 3$, where the scale factor increases as $a(t) \sim e^{t^{2 / 3}}$. Also, we noted that in the limit $\alpha \rightarrow 0$, the scalar spectral index $n_{s}$, given by Eq.(21), coincides with that corresponding to intermediate-inflationary model, where $n_{s}=1-C_{1} / \phi^{2}$ with $C_{1}=8(1-f)(2-3 f) / f^{2}$, see Ref. [9].

The scalar spectral index $n_{s}$ in terms of the number of e-folds $N$, becomes $n_{s} \simeq 1-\frac{2-3 f}{1+f(N-1)}-\left[\frac{\alpha_{0} \beta \gamma}{A f(1+\beta)}\right]\left[1-\alpha_{0}([1+f(N-1)] / A f)^{\gamma / f}\right]^{-1}\left[\frac{1+f(N-1)}{A f}\right]^{(\gamma-f) / f}$. 
On the other hand, the generation of tensor perturbations during the scenario inflationary would produce gravitational wave [29]. The corresponding spectrum is

$$
\mathcal{P}_{g}=8 \kappa\left(\frac{H}{2 \pi}\right)^{2}=\frac{2 \kappa}{\pi^{2}} A^{2} f^{2}\left(\mathcal{B}^{-1}[K \phi]\right)^{-2(1-f)} .
$$
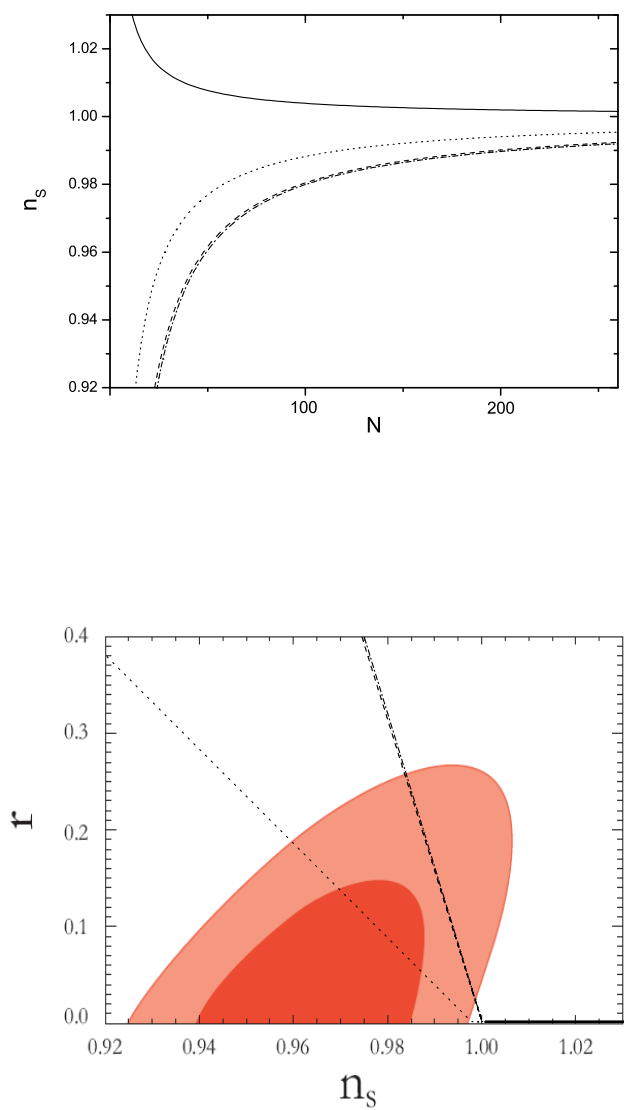

FIG. 1: The upper panel shows the evolution of the scalar spectrum index $n_{s}$ versus the number of e-folds $N$. The lower panel shows the contour plot for the parameter $r$ as a function of the $n_{s}$ at lowest order, for the case of the standard field. Here, from WMAP seven-years data[3], twodimensional marginalized constraints (68\% and 95\% confidence levels) on inflationary parameters $r$ and $n_{s}$. Dotted, dashed, solid and dot-dashed lines are for the pairs $(\alpha=0.81, \beta=0.2)$, $(\alpha=0.775, \beta=0.00126),(\alpha=0.85, \beta=0.6)$, and the standard intermediate model $(\alpha=0)$, respectively. In both panels we have taken the values $\rho_{C h 0}=1, f=1 / 2, \kappa=1$ and $A \simeq$ $2.635 \times 10^{-2} ; 8.225 \times 10^{-2} ; 8.407 \times 10^{-5}$, respectively.

An important observational quantity is the tensor to scalar ratio $r$, which is defined as 
$r=\left(\frac{\mathcal{P}_{g}}{P_{\mathcal{R}}}\right)$. From Eqs.(19) and $(\underline{23})$ we write the tensor to scalar ratio as

$$
r(\phi) \simeq \frac{16(1-f)}{A f\left(\mathcal{B}^{-1}[K \phi]\right)^{f}}\left[1-\alpha\left(\frac{\kappa}{3 A^{2} f^{2}}\right)^{1+\beta}\left(\mathcal{B}^{-1}[K \phi]\right)^{2(1-f)(1+\beta)}\right]^{\frac{-\beta}{1+\beta}}
$$

Combining Eqs.(11) and (24), we can write the tensor-scalar ratio $r$ in terms of the number $N$, as

$$
r(N) \simeq \frac{16(1-f)}{1+f(N-1)}\left[1-\alpha\left(\frac{\kappa}{3 A^{2} f^{2}}\right)^{1+\beta}\left[\frac{1+f(N-1)}{A f}\right]^{\frac{2(1-f)(1+\beta)}{f}}\right]^{\frac{-\beta}{1+\beta}} .
$$

In Fig.(11), the upper panel shows the evolution of the scalar spectrum index $n_{s}$ versus the number of e-folds $N$, and the lower panel shows the contour plot for the parameter $r$ as a function of the $n_{s}$ at lowest order, for different values of the parameters-GCG, $\alpha$ and $\beta$ in the case of the standard scalar field. In particular, the dotted, dashed, solid and dotdashed lines are for the pairs $(\alpha=0.81, \beta=0.2)$ see Ref.[17], $(\alpha=0.775, \beta=0.00126)[18]$, ( $\alpha=0.85, \beta=0.6)[17]$, and the standard intermediate model $(\alpha=0)[9]$, respectively. Here, we have used the value $\rho_{C h 0}=1$, then the parameter $B_{s}=\alpha / \rho_{C h 0}^{1+\beta}=\alpha$. From the upper panel, we noted that the $n_{s}$ graphs for the pair $(\alpha=0.775, \beta=0.00126)$ present a small displacement with respect to the number of e-folds $N$, when compared to the results obtained in the standard intermediate model, in which $\alpha=0$.

On the other hand, from Ref.[3], two-dimensional marginalized constraints (68\% and 95\% confidence levels) on inflationary parameters $r$ and $n_{s}$, the spectral index of fluctuations, defined at $k_{0}=0.002 \mathrm{Mpc}^{-1}$. In order to write down values that relate the tensor to scalar ratio and the spectral index we numerically solved Eqs. (21) and (24). Also, we have used the values $f=1 / 2, \kappa=1$ and for the parameter $A$ the values $A \simeq 2.635 \times 10^{-2} ; 8.225 \times$ $10^{-2} ; 8.407 \times 10^{-5}$, respectively. We noted that the pairs $(\alpha=0.81, \beta=0.2)$ and $(\alpha=0.775$, $\beta=0.00126$ ), the model is well supported by the data as could be seen from Fig.(11). Also, we noted that the pair ( $\alpha=0.85, \beta=0.6$ ) given by solid line, becomes disfavored from observational data, since the spectral index $n_{s}>1$. Also, we noted that for this pair $r \sim 0$ (solid line). We have found that the pair $(\alpha=0.775, \beta=0.00126)$, present a small displacement in relation to the standard intermediate model that corresponds to $\alpha=0$, as could be seen from the Fig.(1).

In this way, we have shown that the intermediate-GCG inflationary model is less restricted 
than analogous ones standard intermediate inflationary models due to the introduction of new parameters, i.e., $\alpha$ and $\beta$ parameters.

\section{B. Tachyon field}

For a tachyonic field the power spectrum of the curvature perturbations is given by $\mathcal{P}_{R}=\left(\frac{H^{2}}{2 \pi \phi}\right)^{2} \frac{1}{Z_{S}}$ [33], where $Z_{S}=V\left(1-\dot{\phi}^{2}\right)^{-3 / 2} \approx V$ [34]. Following Ref.[34], the power spectrum $\mathcal{P}_{R}$ is approximated to be $\mathcal{P}_{R} \simeq\left(\frac{H^{2}}{2 \pi \phi}\right)^{2} \frac{1}{V}$. From Eq.(13) and considering Eq.(15), we write the power spectrum in terms of the tachyonic field in the following way

$$
\mathcal{P}_{\mathcal{R}} \simeq \frac{\kappa}{8 \pi^{2}} \frac{(A f)^{3}}{(1-f)}\left(\widetilde{\mathcal{B}}^{-1}[\widetilde{K} \phi]\right)^{3 f-2}\left[1-\alpha\left(\frac{\kappa}{3(A f)^{2}}\right)^{1+\beta}\left(\widetilde{\mathcal{B}}^{-1}[\widetilde{K} \phi]\right)^{2(1+\beta)(1-f)}\right]^{\frac{\beta}{1+\beta}} .
$$

The scalar spectral index $n_{s}$, using Eq.(15), is given by

$$
n_{s} \simeq 1-\frac{2-3 f}{A f\left(\widetilde{\mathcal{B}}^{-1}[\widetilde{K} \phi]\right)^{f}}-\left[\frac{\alpha_{0} \beta \gamma}{A f(1+\beta)}\right]\left[1-\alpha_{0}\left(\widetilde{\mathcal{B}}^{-1}[\widetilde{K} \phi]\right)^{\gamma}\right]^{-1}\left(\widetilde{\mathcal{B}}^{-1}[\widetilde{K} \phi]\right)^{\gamma-f}
$$

Again, as the case of the standard scalar field from Eq.(27), we see that $n_{s} \neq 1$, for the case $f=2 / 3$.

On the other hand, the amplitude of tensor perturbations $\mathcal{P}_{g}$, is given by

$$
\mathcal{P}_{g}=8 \kappa\left(\frac{H}{2 \pi}\right)^{2}=\frac{2 \kappa}{\pi^{2}} A^{2} f^{2}\left(\widetilde{\mathcal{B}}^{-1}[\widetilde{K} \phi]\right)^{-2(1-f)} .
$$

From expressions (26) and (28) we write the tensor to scalar ratio as

$$
r(\phi)=\frac{16(1-f)}{A f}\left(\widetilde{\mathcal{B}}^{-1}[\widetilde{K} \phi]\right)^{-f}\left[1-\alpha\left(\frac{\kappa}{3(A f)^{2}}\right)^{1+\beta}\left(\widetilde{\mathcal{B}}^{-1}[\widetilde{K} \phi]\right)^{2(1+\beta)(1-f)}\right]^{-\frac{\beta}{1+\beta}}
$$

Again, we noted that when $\alpha \rightarrow 0$ and considering Eqs.(27) and (29) the consistency relations

at lowest order, $n_{s}=n_{s}(r)$, reduced to standard tachyonic model, where $n_{s}=1-\frac{2-3 f}{16(1-f)} r$, see Ref.[26].

We noted numerically from Eqs.(27) and (29) that the trajectories in the $n_{s}-r$ plane between standard field and tachyon field can not be distinguished at lowest order. This coincidence in the consistency relations, $n_{s}=n_{s}(r)$ between standard field and tachyon field, has already been noted in Ref.[35]. Nevertheless, the tachyon field inflationary leads 
to a deviation at second order in the consistency relations, where the spectral index at second order $n_{s}^{(2)}$, becomes [35]

$$
n_{s}^{(2)} \approx-\left(2 \varepsilon^{2}+2\left[2 C_{1}+3-2 C_{2}\right] \varepsilon \eta+2 C_{1} \eta \gamma\right)
$$

where the product $\eta \gamma=\left(9 m_{p}^{4} / 2\right)\left[2 V^{\prime \prime} V^{\prime} / V^{4}-10 V^{\prime \prime} V^{\prime 2} / V^{5}+9 V^{\prime 4} / V^{6}\right]$, the constant $C_{1}$ is a numerical constant approximately $C_{1} \simeq-0.72$ and the constant $C_{2}$; is $C_{2}=0$ in the case of the standard scalar field and $C_{2}=1 / 6$ for tachyon field, respectively. Following Ref.[35], the expression for the tensor to scalar ratio at second order $r^{(2)}$, in the tachyon field is given by

$$
r^{(2)} \approx 16 \varepsilon\left(2 C_{1} \eta-2 C_{2} \varepsilon\right)
$$

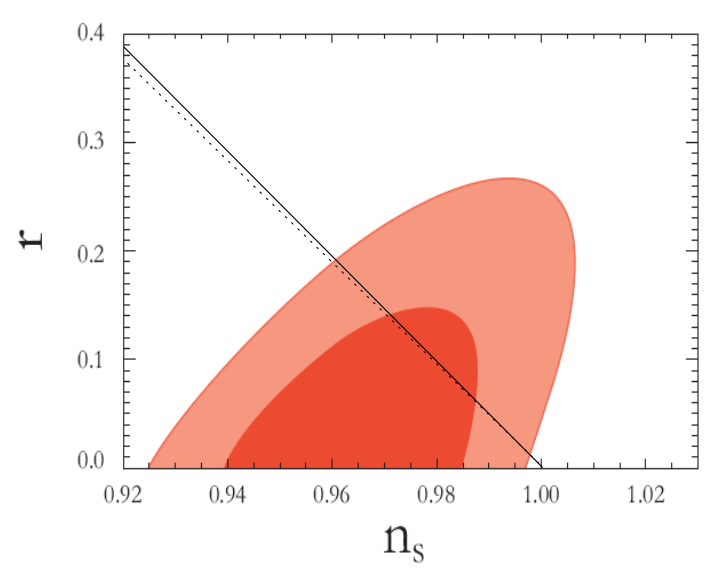

FIG. 2: Contour plot for the parameter $r$ as a function of the $n_{s}$ for the pair $(\alpha=0.81, \beta=0.2)$ in the case of the tachyonic field. Solid and dotted lines are for the trajectories at lowest order and at second order, respectively. Again, as before in drawing the graphs we took $A \simeq 2.635 \times 10^{-2}$, $\rho_{C h 0}=1, f=1 / 2$ and $\kappa=1$.

In Fig.(2), we show the dependence of the tensor to scalar ratio $r$ on the spectral index $n_{s}$, for the pair $(\alpha=0.81, \beta=0.2)$ in the case of the tachyonic field. Solid and dotted lines are 
for the trajectories at lowest order and at second order, respectively. In order to write down values that relate the tensor to scalar ratio and the spectral index, we numerically solved Eqs.(27), (29), (30) and (31). Again as before, we have used the values $A \simeq 2.635 \times 10^{-2}$, $\rho_{C h o}=1, f=1 / 2$ and $\kappa=1$. We observed numerically, that the trajectories in the $n_{s}-r$ plane for the tachyonic field, when we used the second-order corrections to our analysis at first-order in slow roll, are small and this correction can be neglected to a very good approximation.

\section{CONCLUSIONS}

In this paper we have investigated the intermediate inflationary model in GCG. In the slow-roll approximation we have found solutions of the Friedmann equations for a flat universe containing a standard scalar field or a tachyonic field, respectively. In particular, for both scalar fields and from the scenario of intermediate inflation, we have obtained explicit expressions for the corresponding, effective potential, power spectrum of the curvature perturbations, tensor to scalar ratio and scalar spectrum index.

For the scalar field, we have considered the constraints on the parameters of the GCG, from the WMAP seven year data. Here, we have taken the constraint $r-n_{s}$ plane at lowest order in the slow roll approximation. In order to write down values that relate the tensor to scalar ratio and the spectral index we numerically solved Eqs. (21) and (24). We noted that the pairs $(\alpha=0.81, \beta=0.2)$ and $(\alpha=0.775, \beta=0.00126)$, the model is well supported by the data as could be seen from Fig.(1). Also, we noted that the pair $(\alpha=0.85, \beta=0.6)$ given by solid line, becomes disfavored from observational data, since the spectral index $n_{s}>1$. We have found that the pair $(\alpha=0.775, \beta=0.00126)$, present a small displacement in relation to the standard intermediate model that corresponds to $\alpha=0$, as could be seen from the Fig.(11). In particular, we have used the values $\rho_{C h 0}=1, f=1 / 2, \kappa=1$ and $A \simeq 2.635 \times 10^{-2} ; 8.225 \times 10^{-2} ; 8.407 \times 10^{-5}$, respectively.

For the tachyonic field, we noted numerically from Eqs.(27) and (29) that the trajectories in the $n_{s}-r$ plane between standard field and tachyon field can not be distinguished at lowest order. However, we have obtained the dependence of the tensor to scalar ratio $r$ on the spectral index $n_{s}$ at second order. In order to write down values that relate the tensor to scalar ratio and the spectral index at second order, we numerically solved Eqs.(27), (29), 
(30) and (31), for the pair $(\alpha=0.81, \beta=0.2)$. In this case, we observed numerically that the trajectories in the $n_{s}-r$ plane, when we used the second-order corrections to our analysis with respect to the first-order corrections in slow roll are small, as can be seen from Fig.(2).

Finally, we have shown that the intermediate-GCG inflationary models are less restricted than analogous ones standard intermediate inflationary models due to the introduction of new parameters, i.e., $\alpha$ and $\beta$ parameters. The incorporation of these parameters gives us a freedom that allows us to modify the standard intermediate model by simply modifying the corresponding values of the parameters $\alpha$ and $\beta$.

\section{Acknowledgments}

R.H. was supported by COMISION NACIONAL DE CIENCIAS Y TECNOLOGIA through FONDECYT grants $\mathrm{N}^{0} 1090613, \mathrm{~N}^{0} 1110230$ and by DI-PUCV grant 123.703/2009. M.O. was supported by Proyecto D.I. PostDoctorado 2012 PUCV. N.V. was supported by Proyecto Beca-Doctoral CONICYT N² 21100261.

[1] A. Guth, Phys. Rev. D 23, 347 (1981).

[2] A. Albrecht and P. J. Steinhardt, Phys. Rev. Lett. 48, 1220 (1982); A complete description of inflationary scenarios can be found in the book by A. Linde, Particle physics and inflationary cosmology (Gordon and Breach, New York, 1990).

[3] D. Larson et al., Astrophys. J. Suppl. 192, 16 (2011).

[4] C. L. Bennett et al., Astrophys. J. Suppl. 192, 17 (2011); N. Jarosik et al., Astrophys. J. Suppl. 192, 14 (2011).

[5] F. Lucchin and S. Matarrese, Phys. Rev. D32, 1316 (1985).

[6] J. D Barrow, Phys. Lett. B 235, 40 (1990); J. D Barrow and P. Saich, Phys. Lett. B 249, 406 (1990);A. Muslimov, Class. Quantum Grav. 7, 231 (1990); A. D. Rendall, Class. Quantum Grav. 22, 1655 (2005).

[7] J. D Barrow and A. R. Liddle, Phys. Rev. D 47, R5219 (1993); A. A. Starobinsky JETP Lett. 82, 169 (2005); S. del Campo, R. Herrera, J. Saavedra, C. Campuzano and E. Rojas, Phys. Rev. D 80, 123531 (2009); R. Herrera and N. Videla, Eur. Phys. J. C 67, 499 (2010); 
R. Herrera and E. San Martin, Eur. Phys. J. C 71, 1701 (2011); R. Herrera and M. Olivares, Mod. Phys. Lett. A 27, 1250101 (2012); R. Herrera and M. Olivares, Int. J. Mod. Phys. D 21, 1250047 (2012).

[8] W. H. Kinney, E. W. Kolb, A. Melchiorri and A. Riotto, Phys. Rev. D 74, 023502 (2006).

[9] J. D. Barrow, A. R. Liddle and C. Pahud, Phys. Rev. D, 74, 127305 (2006).

[10] D. G. Boulware and S. Deser, Phys.Rev. Lett. 55, 2656 (1985); Phys. Lett. B 175, 409 (1986).

[11] T. Kolvisto and D. Mota, Phys. Lett. B 644, 104 (2007); Phys. Rev. D. 75, 023518 (2007).

[12] I. Antoniadis, J. Rizos and K. Tamvakis, Nucl.Phys. B 415, 497 (1994).

[13] S. Mignemi and N. R. Steward, Phys. Rev. D 47, 5259 (1993); P. Kanti, N. E. Mavromatos, J. Rizos, K. Tamvakis and E. Winstanley, Phys. Rev. D 54, 5049 (1996); Ch.-M Chen, D. V. Gal'tsov and D. G. Orlov, Phys. Rev. D 75, 084030 (2007); A. K. Sanyal, Phys. Lett. B, 645,1 (2007).

[14] A. Kamenshchik, U. Moschella and V. Pasquier, Phys. Lett. B 511, 265 (2001).

[15] U. Alam, V. Sahni, T.D. Saini, and A.A. Starobinsky, Mon. Not. R. Astron. Soc. 344, 1057 (2003); L. Amendola, F. Finelli, C. Burigana, and D. Carturan, JCAP 0307, 005 (2003); X. Zhang, F.-Q. Wu, and J. Zhang, JCAP 0601, 003 (2006); L. Xu, J. Lu, JCAP 1003, 025(2010); J. Lu, Y. Gui, L. Xu, Eur. Phys. J. C 63,349(2009); Z. Li, P. Wu, H. Yu, JCAP09,017(2009).

[16] N. Liang, L. Xu, Z. H. Zhu, Astrono. Astrophy, 527, A11(2011); C. G. Park, J. c. Hwang, J. Park, H. Noh, Phys. Rev. D 81,063532(2010).

[17] M. C. Bento, O. Bertolami and A. Sen, Phys. Rev. D 66, 043507 (2002).

[18] L. Xu, J. Lu, Y. Wang, J. Lu and Y. Wang, Eur. Phys. J. C 72, 1883 (2012).

[19] H. B. Benaoum, arXiv:hep-th/0205140; A. Dev, J.S. Alcaniz and D. Jain, Phys. Rev. D 67 023515 (2003); H. Sandvik, M. Tegmark, M. Zaldarriaga and I. Waga, Phys. Rev. D 69, 123524 (2004) ; L. Amendola, I. Waga and F. Finelli, JCAP 0511, 009 (2005); L.P. Chimento, Phys. Rev. D 69, 123517 (2004); L.P. Chimento and R. Lazkoz, Phys. Lett. B 615, 146 (2005); U. Debnath, A. Banerjee and S. Chakraborty, Class. Quant. Grav. 21, 5609 (2004); J.C. Fabris, S.V.B. Goncalves and P.E. de Souza, arXiv:astro-ph/0207430; M. Biesiada, W. Godlowski and M. Szydlowski, Astrophys. J. 622, 28 (2005); Y. Gong, JCAP 0503, 007 (2005); W. Zimdahl and J.C. Fabris, Class. Quant. Grav. 22, 4311 (2005); P. Wu and H. Yu,Class. Quant. Grav. 24, 4661 (2007); L. P. Chimento and M. G. Richarte, Phys. Rev. D 84123507 (2011); Y. D. Xu, Z. G. Huang and X. H. Zhai, Astrophys. Space Sci. 339, 31 (2012). 
[20] O. Bertolami and V. Duvvuri, Phys. Lett. B 640, 121 (2006).

[21] S. del Campo and R. Herrera, Phys. Lett. B 660, 282 (2008); R. Herrera, Gen. Rel. Grav. 41, 1259 (2009);R. Zarrouki and M. Bennai, Phys. Rev. D 82, 123506 (2010).

[22] V. Gorini, A. Y. .Kamenshchik, U. Moschella and V. Pasquier, Phys. Rev. D 69, 123512 (2004).

[23] L. Randall and R. Sundrum, Phys. Rev. Lett. 83, 4690 (1999); T. Shiromizu, K. Maeda and M. Sasaki, Phys. Rev. D 62, 024012 (2000); R. Maartens, Lect. Notes Phys. 653213 (2004); A. Lue, Phys. Rept. 423, 1 (2006).

[24] S. del Campo and R. Herrera, Phys. Lett. B 665, 100 (2008); R. Herrera, Phys. Lett. B 664, 149 (2008); S. del Campo and R. Herrera, Phys. Lett. B 660, 282 (2008); X. Zhang, J. -f. Zhang, J. -l. Cui and L. Zhang, Mod. Phys. Lett. A 24, 1763 (2009).

[25] A. Prudnikov, Y. Brychkov, and O. Marichev, "More Special Functions", Gordon and Breach Science Publisher, (1990); K. Roach, "Hypergeometric Function Representation", in Proceedings of ISSA 96, ACM, 301-308 (New York, 1996).

[26] S. del Campo, R. Herrera and A. Toloza, Phys. Rev. D 79, 083507 (2009).

[27] J. M. Bardeen, P. J. Steinhardt and M. S. Turner, Phys. Rev. D 28, 679 (1983).

[28] J. Martin and D. J. Schwarz, Phys. Rev. D 57, 3302 (1998); K. A. Malik and D. Wands, Phys. Rept. 475, 1 (2009).

[29] B. A. Bassett, S. Tsujikawa and D. Wands, Rev. Mod. Phys. 78, 537 (2006).

[30] D. Wands, K. A. Malik, D. H. Lyth and A. R. Liddle, Phys. Rev. D 62, 043527 (2000).

[31] R. Zarrouki and M. Bennai, Phys. Rev. D 82, 123506 (2010)

[32] A. Liddle and D. Lyth, Cosmological inflation and large-scale structure, 2000, Cambridge University;J. Linsey, A. Liddle, E. Kolb and E. Copeland, Rev. Mod. Phys 69, 373 (1997).

[33] J. C. Hwang and H. Noh, Phys. Rev. D 66, 084009 (2002).

[34] M. R. Garousi, M. Sami and S. Tsujikawa, Phys. Rev. D 70, 043536 (2004); S. Panda, M. Sami and S. Tsujikawa, Phys. Rev. D 73, 023515 (2006).

[35] D. A. Steer and F. Vernizzi, Phys. Rev. D 70, 043527 (2004). 